\title{
FRM (Fuzzy Relational Maps) Model of Hypertension Problem Faced by Adult in Tamilnadu
}

\author{
A.Divya \\ Research Scholar \\ Department of Mathematics, \\ B.S.Abdur Rahman University Chennai-600 048 \\ Tamilnadu, India
}

\author{
P.S.Sheik Uduman, Ph.D \\ Professor, \\ Department of Mathematics, \\ B.S.Abdur Rahman University Chennai-600 048 \\ Tamilnadu, India
}

\begin{abstract}
Nowadays due to the lifestyle modifications, Hypertension (HP) is commonly found in large number of peoples in Tamilnadu. A similar kind of research has been carried out to examine the living experience of diabetic people in A.Victor Devadoss et.al [1]. In this paper the relation between risk factor and symptoms of hypertension using fuzzy relational map (FRM) in order is derived. Based on this study the major risk factor of hypertension and the preventing measure using it is determined.
\end{abstract}

Keywords: Fuzzy relational maps (FRMs), Risk factors, Symptoms, Hypertension.

\section{INTRODUCTION}

In W.B.Vasantha et.al $[2,3,4]$ a new notion called Fuzzy Relational Maps (FRMs) was introduced. To define a FRM a domain space and a range space which are disjoint in the sense of concepts is needed. The number of elements in the range space need not in general be equal to the number of elements in the domain space. In FRMs the very causal associations is divided into two disjoint units for example the relationship between a teacher and a student or relation between doctor and patient and so on. Further it is assumed that no intermediate relations exist within the domain element or node and the range space. Hypertension can be defined as a blood carried from the heart to all parts of the body in the vessels. Each time the heart beats it pumps blood into the vessels (arteries) as it pumped by the heart. Blood pressure measurements in adults are classified into the four categories: 1.Normal blood pressure less than 120/80, 2. Pre-hypertension 120-139/80-89, 3.High blood pressure (stage 1) 140-159/9099 and 4. High blood pressure (stage 2) higher than 160/100. FRM model is used to deduct the major risk factor for the hypertension people in urban area Clinical guideline 34[5]. Hypertension is the major risk factor for cardio vascular diseases Roger VL et al [6].

\subsection{Fuzzy relational maps (FRMs)}

In Fuzzy relational maps (FRMs) the elements of the domain space are taken from the real vector space of dimension $\mathrm{n}$ and that of the range space are real vectors from the vector dimension $m$ ( $m$ in general need not be equal to $n$ ). Denote $R$ as the set of nodes $R_{1}, \ldots, R_{m}$ of the range space, where $\mathrm{R}=\left\{\left(\mathrm{X}_{1}, \ldots, \mathrm{X}_{\mathrm{m}}\right) / \mathrm{X}_{\mathrm{j}}=0\right.$ or 1$\}$ for $\mathrm{j}=1,2, \ldots, \mathrm{m}$. if $\mathrm{X}_{\mathrm{i}}=1$ it means that the node $R_{i}$ is in the ON state and if $X_{i}=0$ it means that the node $R_{i}$ is in the OFF state. Similarly $D$ denotes the nodes $D_{1}$, $D_{2, \ldots,} D_{n}$ of the domain space, where $D=\left\{\left(X_{1}, \ldots, X_{n} / X_{j}=0\right.\right.$ or 1) for $i=1,2, \ldots, n$ if $X_{i}=1$ it means that the node $D_{i}$ is in the ON state and if $X_{i}=0$ it means that the node $D_{i}$ is in the OFF state W.B.Vasantha et.al[7].

\section{Definition 1.1.1:}

Let $D_{i} R_{j}\left(\right.$ or $\left.R_{j} D_{i}\right), 1 \leq j \leq m, 1 \leq i \leq n$.when $R_{i}\left(\right.$ or $\left.D_{j}\right)$ is switched on and if causality flows through edges of the cycle and if it again causes $R_{i}$ (or $D_{j}$ ) we say that the dynamical system goes round and round. This is true for any node $R_{j}\left(\right.$ or $D_{i}$ ) for $1 \leq \mathrm{i} \leq \mathrm{n}$, (or $1 \leq \mathrm{j} \leq \mathrm{m}$ ) the equilibrium state of this dynamical system is called the hidden pattern.

\section{Definition 1.1.2:}

If the equilibrium state of a dynamical system is a unique state vector, then it is called a fixed point. Consider an FRM with $R_{1}, R_{2}, \ldots, R_{m}$ and $D_{1}, D_{2}, \ldots, D_{n}$ as nodes for example, let us start the dynamical system by switching 0on R1(or D1).let us assume that the FRM settles down with $R_{1}$ and $R m$ (or $D_{1}$ and $\left.D_{n}\right)$ ON i.e. the state vector remains as $((1,0, \ldots, 0,1)$ in $R)$ or $((1,0, \ldots, 0,1)$ in $\mathrm{D})$.this state vector is called the fixed point

\section{Definition 1.1.3:}

If the FRM settles down with a state vector repeating in the form $\quad \mathrm{A}_{1} \rightarrow \mathrm{A}_{2} \rightarrow \mathrm{A}_{3} \rightarrow \ldots \rightarrow \mathrm{A}_{\mathrm{i}} \rightarrow \mathrm{A}_{1}\left(\right.$ orB $\left._{1} \rightarrow \mathrm{B}_{2} \rightarrow \ldots \rightarrow \mathrm{B}_{\mathrm{i}} \rightarrow \mathrm{B}_{1}\right)$ then this equilibrium is called a limit cycle

\section{Methods of determining the hidden pattern}

Let $R_{1}, R 2 \ldots R_{m}$ and $D_{1}, D 2 \ldots D_{n}$ be the nodes of a FRM with feedback. Let $\mathrm{E}$ be the relational matrix. Let us find a hidden pattern when $D_{1}$ is switched on i.e. When an input is given as vector $A_{1}=(1,0 \ldots 0)$ in $D_{1}$, the data should pass through the relational matrix. This is done by multiplying $A_{1}$ with the relational matrix E. Let $\mathrm{A}_{1} \mathrm{E}=\left(\mathrm{r}_{1}, \mathrm{r}_{2} \ldots \mathrm{r}_{\mathrm{m}}\right)$, after thersholding and updating the resultant vector we get $A_{1} E \varepsilon R$.Now let $\mathrm{B}=\mathrm{A}_{1} \mathrm{E}$, we pass on $\mathrm{B}$ into $\mathrm{E}^{\mathrm{T}}$ and obtain $\mathrm{BE}^{\mathrm{T}}$. We update and threshold the vector $\mathrm{BE}^{\mathrm{T}}$ so that $\mathrm{BE}^{\mathrm{T}} \varepsilon \mathrm{D}$. This procedure is repeated till we get a limit cycle or a fixed point. Kosko Bart et .al [8], [9].

\subsection{Risk factors for developing high blood pressure}

The Older the age of a person greater is the likelihood that they will develop high blood pressure, especially elevated systolic readings. This is largely due to arteriosclerosis or "hardening of the arteries "WHO [10]. At younger ages, women are less likely to develop high blood pressure than men. Consuming excess alcohol per day tends to raise blood pressure which is discussed Gary D.Friedman.et al [11] and Arthur L.Klatsky.et al[15]. Poor exercise tolerance and obesity all of which have implicated in the development of high blood pressure. Smoking is powerful risk factors for 
many different human diseases. Some people have high sensitivity to sodium (salt) and their blood pressure increases if they use salt. Reducing sodium intake tends to lower their blood pressure. Hormone imbalances, certain anatomic abnormalities tumors and other medical problems can cause a type of high blood pressure can cause a type of high blood pressure known as secondary hypertension all are WHO[10]

Also having a family history of high blood pressure has higher risk category than someone with no family history of high blood pressure in Winnicki M. et al [12]. Also as body weight increases the blood pressure rises. Obesity is defined as a Body Mass Index (BMI) being overweight increases the risk of high blood pressure. Health care practitioners recommend that all obese people with blood pressure lose weight until they are within $15 \%$ of their healthy body weight is in Clinical Guidelines [13].

Stress especially at a high level has a correlated with increased physical ailments, including cardio vascular issues report published by the WHO titled social determinants of health the report explains how long term stress can make a person more vulnerable to a wide range of conditions including hypertension WHO[10].

\subsection{Symptoms of hypertension}

According to research studies, the risk of dying of a heart attack is directly linked to high blood pressure particularly systolic hypertension. High blood pressure usually causes no symptoms and high blood pressure often labeled" the silent killer" people who have high blood pressure typically don't know it until their blood pressure is measured sometimes people with markedly elevated blood pressure may develop

Headache, dizziness, blurred vision, nausea, vomiting, chest pain, shortness of breath, People often do not seek medical care until they have no symptoms arising from organ damage caused by chronic high blood pressure the organ damage are commonly seen in chronic high blood pressure WHO[10].

\subsection{Facts about hypertension in adult}

Hypertension is a major public health problem in India. Hypertension affects approximately one billion people worldwide. One in three adults worldwide has raised blood pressure. Hypertension is major risk factor for stroke and heart diseases. It is considered directly responsible for 7.5 million deaths in 2004 almost 13\% of all global death. Hypertension is known problem in Tamilnadu, although accurate statistics and more information are available in urban areas like Chennai. The Chennai rural epidemiology study helped to determine the prevalence awareness and control rates of hypertension in Chennai. The overall population prevalence of hypertension was found to be $20 \%$ with $23.2 \%$ of men and $17.1 \%$ of women affected Mohan .V [14].

\section{BASIC MODEL}

The relational between the risk factors and the symptoms of diabetes among adults using fuzzy relational maps A. Victor Devadoss et.al [1], Diabetes is a problem body fuel system which is Cause due to lack of insulin hormone in the pancreases that is essential for getting energy from food causes of diabetes continues to be a mystery till now it is both genetic and environmental factors. The main causes may be due to genetics and environmental factors such as obesity. The study shows that diabetes patients increase in the urban population because the low amount of physical activity in the urban population in Chennai. This leads to increasing urbanization tends to lower the physical activity worldwide.

\section{MODEL: IMPLEMENTATION OF FRM MODEL IN STUDY OF HYPERTENSION PROBLEM}

100 patients were interviewed using a linguistic questionnaire regarding hypertension for a period of one month in the Spot hospital (Chennai). The fuzzy concepts, attributes are first given in the form of matrix relational equations and then solved. The patients who have been investigated reported the symptoms of Hypertension. FRM model is used to find the major risk factor for hypertension. The survey was based on the following questions

\subsection{Questions for the outpatients respondents}

$>$ Whether your smoker or nonsmoker if so how many times do you smoke per day

$>$ Is any of your family members is having hypertension

$>$ Whether alcoholic or non-alcoholic, if so how much do you drink per day

$>$ Are you vegetarian or non vegetarain,what's your food habit, Do you eat fast food

$>$ Do you do proper exercise daily, what is a nature of workout

$>$ Have you reduce the intake of salt level as prescribed by doctor

$>$ How long have you been suffering from stress, have you consulted with doctor

$>$ Have you undergone any surgery, do you have any hormone imbalance problem

$>$ How old are you, when and where you diagnosed, Did you consult a doctor, Are you medications for hypertension

\subsection{Attributes related to the Risk factors}

The domain space $\mathrm{R}$ connected with the risk factors are given by $R=\left\{R_{1}, R 2 \ldots R_{10}\right\}$
R1: Alcohol
R2: Age
R3: Stress
R4: Being overweight
R5: Sodium salt
R6: Smoking
R7: Having a family history
R8: Activity level
R9: Gender
R10: Other medical problems

\subsection{Attributes related to the symptoms}

The Range space $\mathrm{S}$ connected with the symptoms are given by $\mathrm{S}=\left\{\mathrm{S}_{1}, \mathrm{~S} 2 \ldots \mathrm{S} 7\right\}$

\section{$\mathrm{S}_{1}:$ Headache}

$\mathrm{S}_{2}$ : Dizziness

$\mathrm{S}_{3}$ : Nausea

$\mathrm{S}_{4}$ : Chest pain

$\mathrm{S}_{5}$ : Blurred vision

$\mathrm{S}_{6}$ : Shortness of breath

$\mathrm{S}_{7}$ : Vomiting 


\subsection{Diagrammatic representation of FRM}

Figure 1. FRM

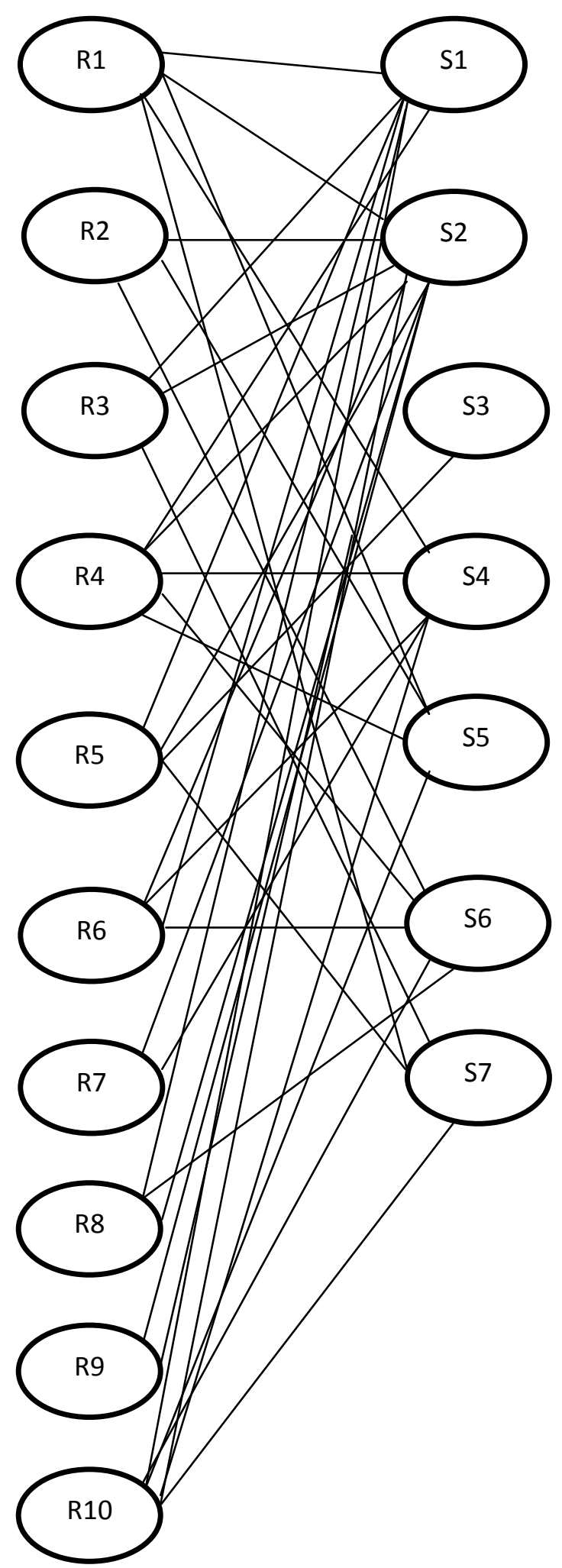

\subsection{First Expert's Opinion}

The opinion of the first expert is a hypertension from urban and is given vital importance. This opinion is transformed into the fuzzy relational matrix $\mathrm{M}_{1}$ is given by

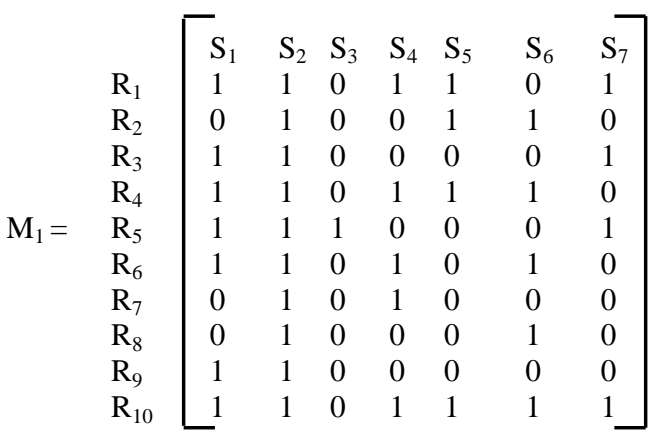

The hidden pattern of the state $X=\left(\begin{array}{llllllllll}0 & 0 & 0 & 1 & 0 & 0 & 0 & 0 & 0 & 0\end{array}\right)$ is obtained by the following method

$$
\begin{aligned}
& \mathrm{XM}_{1} \hookrightarrow\left(\begin{array}{lllllll}
1 & 1 & 0 & 1 & 1 & 1 & 0
\end{array}\right)=\mathrm{Y} \\
& \mathrm{YM}_{1} \hookrightarrow\left(\begin{array}{llllllllll}
1 & 1 & 1 & 1 & 1 & 1 & 1 & 1 & 1 & 1
\end{array}\right)=\mathrm{X}_{1} \\
& \mathrm{X}_{1} \mathrm{M}_{1} \hookrightarrow\left(\begin{array}{lllllll}
1 & 1 & 1 & 1 & 1 & 1 & 1
\end{array}\right)=\mathrm{Y}_{1}
\end{aligned}
$$

(Where $\hookrightarrow$ denotes after thresholding and updating the resultant vector)

When only the nodes $\mathrm{R}_{4}$ Obesity in the $\mathrm{ON}$ state and all other attributes to be in the OFF state then all the nodes in the domain space come to ON state. It is seen that the effect of $\mathrm{X}$ on the dynamical system $\mathrm{M}$ is a fixed point given by the binary pair $\{(1111111111)(1111111)\}$. When having obesity node alone in the ON state and let $\mathrm{X}=(1111111)$, then the resultant is the fixed point given by the binary pair $\{(1111111111)$ (1111111)\}.When ON state is taken as node $\mathrm{R}_{4}$. It is seen that the hidden pattern is the fixed point. This is the same binary pair which makes all the nodes to be in the ON state in the Domain Space and also it makes all the nodes in the range space to be in $\mathrm{ON}$ state.

Figure 2: Alcohol Vs symptoms

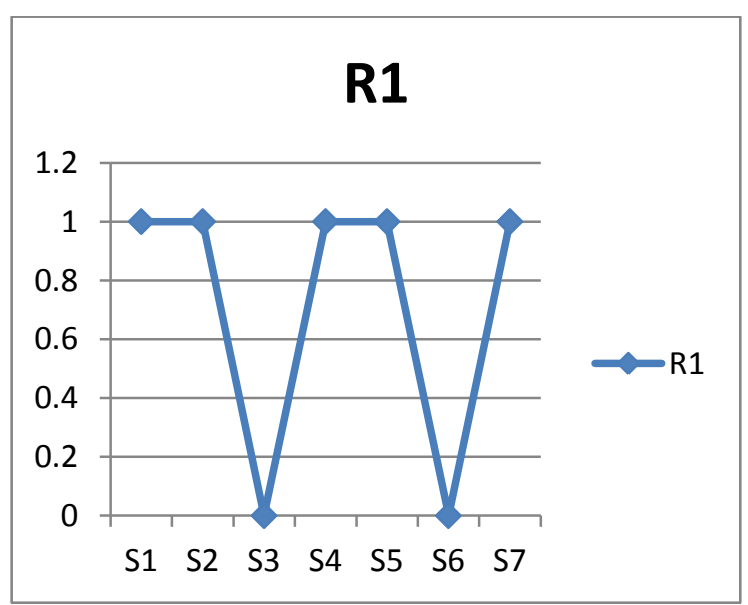


Figure 3: Age Vs symptoms

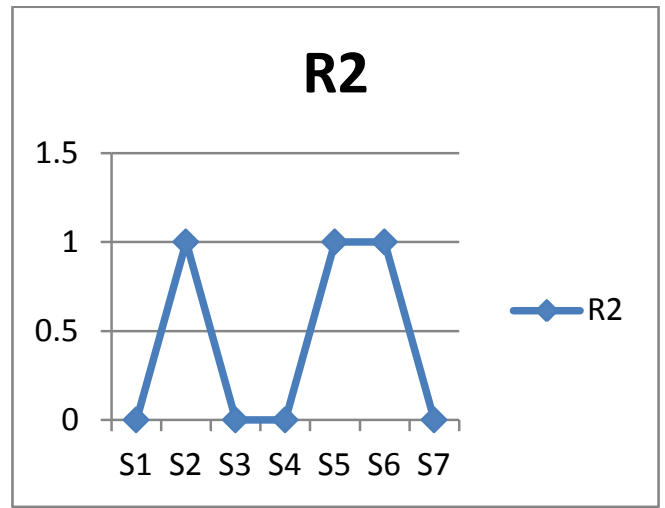

Figure 4: Stress Vs Symptoms

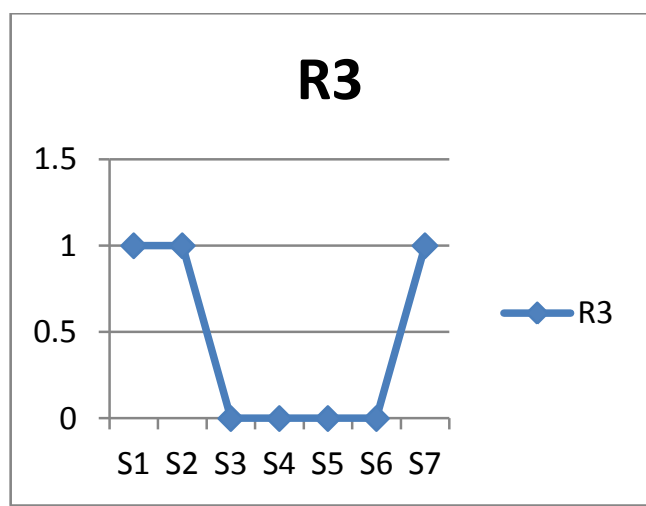

Figure 5: Being overweight Vs Symptoms

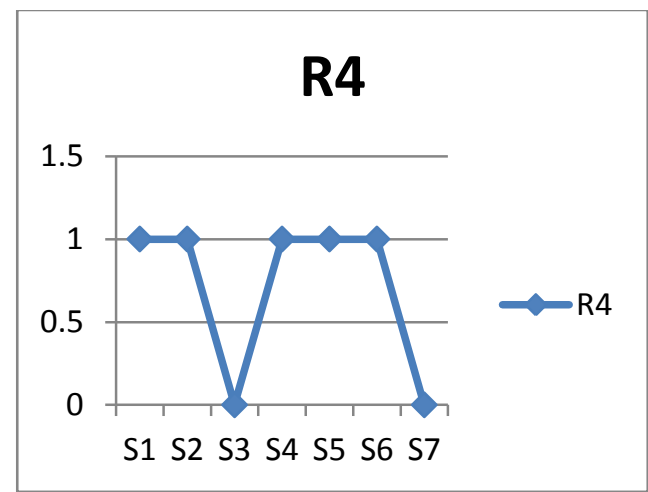

Figure6: Sodiumsalt Vs Symptoms

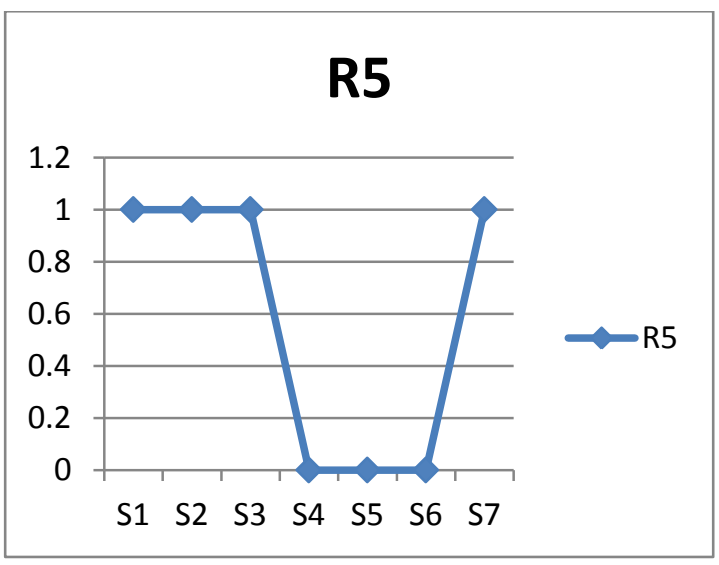

Figure7: Smoking Vs Symptoms

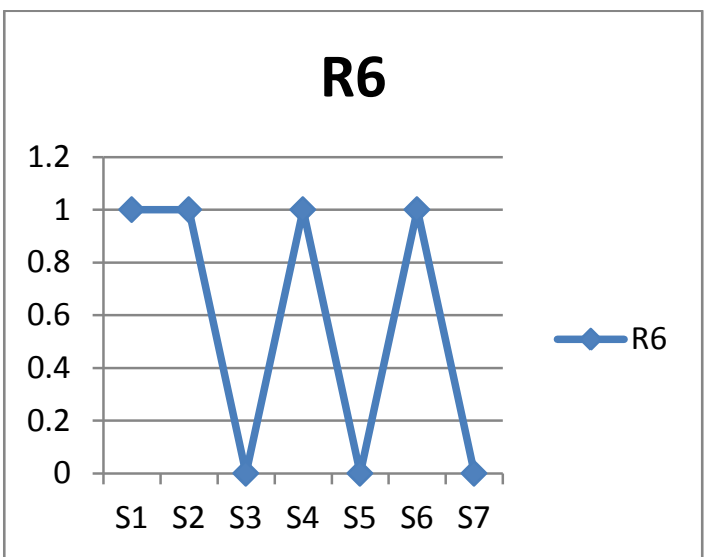

Figure 8:Having family history Vs Symptoms

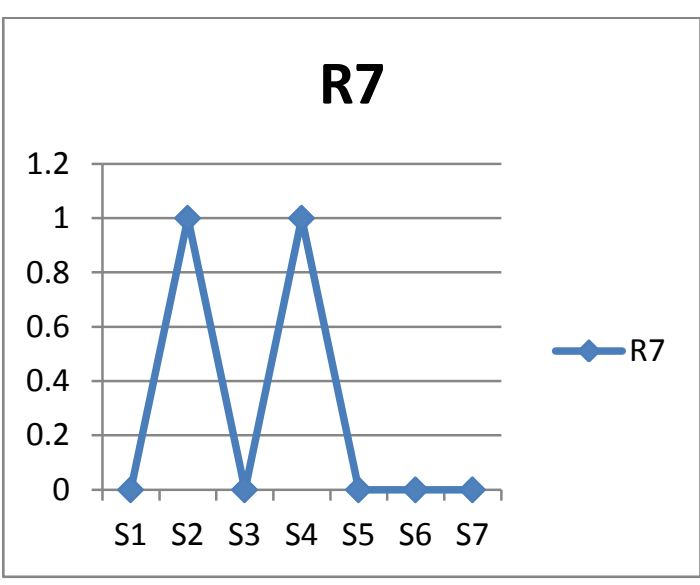

Figure 9:Activity Level Vs Symptoms

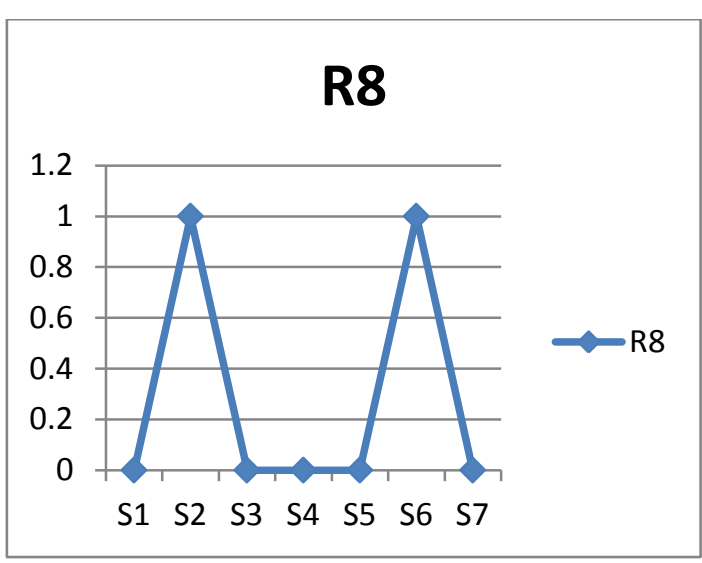


Figure 10: Gender Vs Symptoms

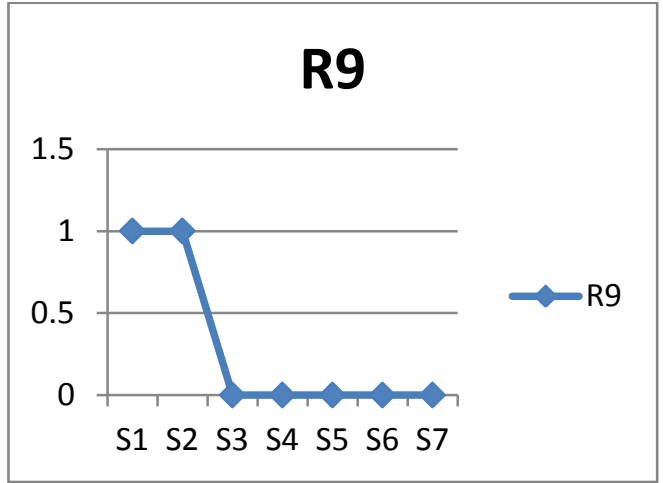

Figure 11: Other medical problems Vs symptoms

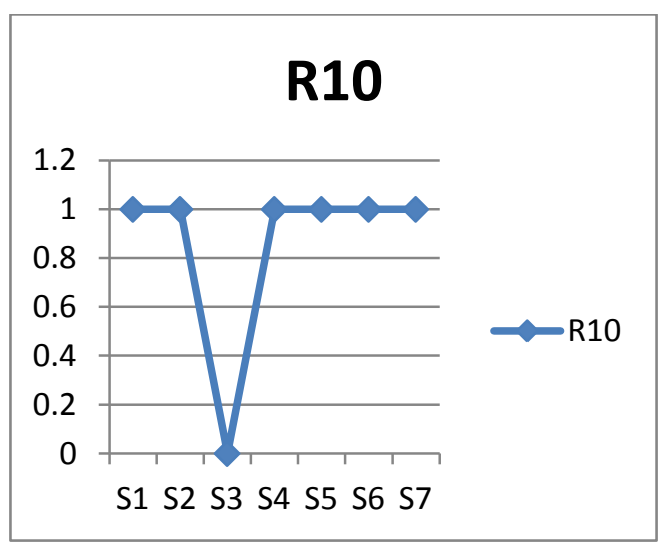

\section{CONCLUSION}

Hypertension is a silent killer and it is the major risk factor for cardiovascular diseases. Based on the graph Alcohol, Being overweight (Obesity), Sodium salt, other medical problems, leads to the risks of hypertension. From the study, it is seen that while using FRM, Being overweight (obesity) is the major risk factor for hypertension.

Hypertension may be prevented by living a healthy lifestyle such as eating a nutritious, low fat diet, exercising regularly, maintaining a healthy weight, quitting alcohol, impede smoking and reduce stress in day to day life.

\section{ACKNOWLEDGEMENT}

The author wishes to thank Dr S.Srinivasan, Dr I. Raja Mohammed and Mrs. Dowlath Fathima, B.S. Abdur Rahman University, Chennai for their instant support.

\section{REFERENCES}

[1] A.Victor Devadoss, V.Susanna Mystica, The living experience of a diabetic adult in India using Fuzzy Relational Maps (FRM), World-comp.org/P2011/BIC 3157.Pdf July 21,2011(the 2011 world congress in computer science,computer engineering and applied computing Las Vegas, Nevada, USA July 18-21.
[2] Vasantha Kandaswamy W.B., and Yasmin sultana knowledge processing using fuzzy relations maps, ultra.sci 12(2000); 242-245.

[3] Yasmin sultana, construction of employer-employee relationship model using fuzzy relational maps master dissertation, guide W.B.Vasantha Kandaswamy, department of mathematics, Indians institute of technology April 2000.

[4] W.B.VasanthaKandaswamy, Florentine smarandache Fuzzy cognitive maps and Neutroscophic cognitive maps.

[5] NICE/BHS clinical guideline 34: hypertension: management of hypertension in adult in primary care: partial update http://www.nice.org.uk/CG034 guidance (accessed June 28, 2006).

[6] Veronique L.Roger, S.Alan, Donald M, Lloyd-jones, Robert J.Adams, Jarett D.Berry,Todd M.Brown, (2010) heart diseases and stroke statistics 2011 update. A report from the American Heart association circulation 123(4):e18-e209.

[7] Vasantha Kandaswamy W.B and Yasmin sultana FRM to analyses the Employee-Employer relationship model. Bihar math.soc.21 (2001); 25-34.

[8] KoskoBart Neural Networks and Fuzzy systems.prentince-hall Englewood cliffs, New Jersy (1992).

[9] KoskoBart, Fuzzy Cognitive Maps, International Journal of Man-Machine studies (1986)34, 65-75.

[10] World Health Organization India mortality country factsheet. Retrived from http:www.who.int/whosis/mort/profiles/mort_searo_ind_ india.pdf (2006).

[11] Gary D.Friedman, Arthur L.Klastsky, A.B.Siegelaub. Alcohol intake and hypertension.ann.int med, 98:846849(1983)

[12] Winnicki M.Somers VK,Dorigatti F,Longo D,Santonastaso M,Mosl Matterei M,Pessina A.C,Palatini $\mathrm{P}$, lifestyle family history and progression of Hypertension, Journal of Hypertension 24(8) 147987(2006).

[13] Clinical guidelines on the identification evaluation and treatment of overweight and obesity in adults-the evidence report national institutes of health.obes.res.1998: 6(suppl2): 51s-2095.pr.

[14] V.Mohan, M.Deepa, S.Farooq, M.Datta and R.Deepa, prevalence awareness and control of hypertension in Chennai-the Chennai urban rural epidemiology study. Journal of the association of physicians of India, 2007, 55(May).

[15] Arthur L.Klatsky, Gary D.Friedman, Abraham B.Siegelaub, Marie.J.Gerard, Alcohol consumption and blood pressure new eng J med, 296:1194-1200(1977). 